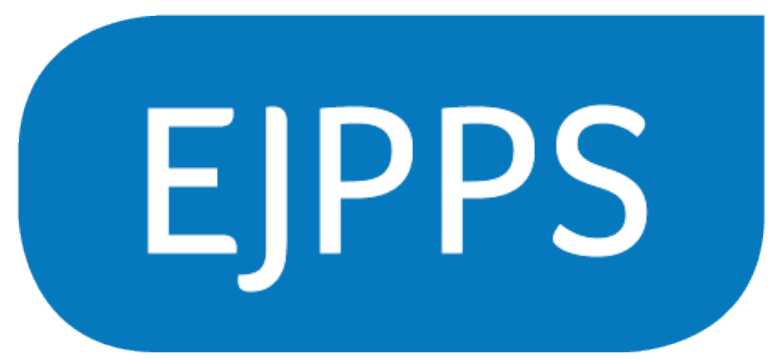

EUROPEAN JOURNAL OF

PARENTERAL AND

PHARMACEUTICAL SCIENCES

EJPPS - European Journal of Parenteral and Pharmaceutical Sciences Volume 26 Issue 3

https://www.ejpps.online/post/vol23-6-microbial-air-samplers-for-meaningful-

cleanroom-environmental-monitoring

https://doi.org/10.37521/ejpps.26301

Microbial Air Samplers for Meaningful Cleanroom Environmental Monitoring

Tim Eaton

Sterile Manufacturing Specialist, AstraZeneca, UK

Corresponding Author: Tim Eaton

AstraZeneca,

UK Operations,

Silk Road Business Park,

Macclesfield

Cheshire. SK10 2NA

England

Email: tim.eaton@astrazeneca.com 


\title{
Microbial Air Samplers for Meaningful Cleanroom Environmental Monitoring
}

Tim Eaton Sterile Manufacturing Specialist, AstraZeneca, UK

\begin{abstract}
Airborne microbiological concentrations within pharmaceutical cleanrooms are determined by sampling and to maximise the detection of any airborne microbes, it is essential that the sampling is undertaken in locations where there is greatest contamination risk using air samplers that have a verified and appropriate performance. Sampler performance can be assessed by review of both the physical and biological collection efficiencies that are determined by testing. The physical collection efficiency is the ability to collect particles of various sizes and the biological collection efficiency assesses the collection of viable microbes that includes the losses caused by the physical collection efficiency and the detrimental effect that the sampling has on the viability of the captured microbes. Due to the limitations of the established biological collection efficiency test method, this efficiency is only determined for microbes of sub-micron size which are not representative of the larger microbecarrying particles typically present with cleanrooms.

Samplers with a low physical collection efficiency for sub-micron particles are likely to have a poor performance when this test method is utilised and, in an attempt, to remove this bias from the testing the 'biological efficiency', is often reported. This is a measure of the likelihood that any captured microbes would survive but is often mistaken for the biological collection efficiency and samplers may be utilised in the false belief that they have an appropriate performance. This article provides information regarding air sampler performance testing and reviews the test results reported by the same independent specialist testing company, therefore negating issues resulting from different testing methods, for three different air samplers. The results that are used to determine the 'biological efficiency' are examined to provide information relating to the biological collection efficiency of each sampler and to also provide additional information relating to the physical collection efficiency. Improvements to enhance the air sampler testing procedures, to enable a better direct comparison of the performance of different samplers, are suggested.
\end{abstract}

Keywords - microbiological air sampler, biological collection efficiency, physical collection efficiency, biological efficiency 


\section{$1 \quad$ INTRODUCTION}

Microbial air sampling is performed in cleanrooms and clean zones to determine the airborne microbial concentrations and for licensed pharmaceutical and healthcare applications, the airborne concentrations limits are specified in the regulatory authority guidelines ${ }^{1}$. The sampling results are utilised to confirm that the specified operational cleanliness levels have been attained and are also likely to be utilised to support the release of any manufactured product. For well controlled cleanrooms, airborne microbial concentrations will be low and for critical clean zones are expected to be effectively zero. The limitations of environmental monitoring to provide comprehensive data that confirms full control of the environment throughout all operational activities is well understood. For example, consider a unidirectional airflow (UDAF) clean zone that is supplied with air at a velocity of $0.45 \mathrm{~m} / \mathrm{s}$ through a $2 \mathrm{~m}^{2}$ entrance plain. For a 4 hour work period, a total of $12960 \mathrm{~m}^{3}$ of air is supplied and a single $1 \mathrm{~m}^{3}$ sample, taken over a 20 minute period, is only $0.008 \%$ of the total volume of the supplied air and represents only $8.3 \%$ of the time of the full work period. With further consideration for the localised nature of UDAF, which does not distribute contamination throughout the environment, this very limited information would be further compromised if the air sampler is located where there is no risk of product or critical surface contamination. A formal risk assessment method, that utilises the correct risk factors that relate to microbial contamination, has been shown elsewhere 2,3 to effectively identify the activities that present the highest risk of contamination, and can be applied to determine the most meaningful operational monitoring locations.

The sampler utilised at the identified locations must have an appropriate and verified performance in order to maximise the effectiveness of the monitoring programme. However, an air sampler that has a poor performance would have limited ability to capture and recover airborne microbes and may incorrectly confirm that the required cleanliness standards have been achieved. The performance of many air samplers is poor and considerably variable with reported collection of the micro-organisms in the environment ranging from about $22 \%$ to over $99 \%{ }^{4}$. The testing requirements for the verification of air sampler performance are discussed further in the following sections.

REQUIREMENTS AND CONSIDERATIONS FOR MICROBIAL AIR SAMPLERS

The regulatory authority expectations are that the sampling method and equipment used should be fully understood and the recovery efficiency of the chosen sampling method should be qualified.

Procedures for sampler validation are detailed in informative Annex E of BS EN $17141^{5}$. (Note; This standard is an update of ISO 14698-1:2003 ${ }^{6}$ and BS EN ISO 14698-2:2003 ${ }^{7}$ which include sampler validation requirements, and has replaced both in Europe and the UK). This validation tests for both the physical and biological collection efficiencies and to assess the overall performance of a sampler, both sets of tests need to be carefully considered as they give very distinct information. Due to the complex activities required, the validation of a sampler is likely to be carried out by an independent specialist testing company. It is expected that the results are provided by the manufacturer of the sampler, and reviewed as part of the assessment to determine if the sampler is appropriate for the intended application. The testing is now considered in further detail.

\section{Physical Collection Efficiency}

The physical collection efficiency relates to the ability of the sampler to collect particles of various sizes and measures the collection of inert particles and is influenced by the air inlet and the separation efficiencies. The air inlet efficiency is a function of the air inlet design and its ability to collect particles from the air in a representative way and transport them to the impaction nozzle. The separation efficiency is the ability of the sampler to separate and collect particles of different sizes from the air stream by impaction onto the collection medium. The physical collection efficiency is the same 
whether the particles are microbe-carrying particles (MCPs), unicellular microbes or non-viable (inanimate) particles and will vary according to the size of the collected particle.

The physical collection efficiency can be determined experimentally and is based on the physical characteristics of the sampling device such as airflow, orifice shape, orifice size and the number of orifices. The $d_{50}$ (cut-off size) describes the aerodynamic or equivalent particle diameter at which $50 \%$ is removed from the airstream and impacted onto the media. A simplified formula to calculate the $d_{50}$ value (in $\mu \mathrm{m})^{8}$ is shown in the equation 1 ;

$$
\mathrm{d}_{50}=\sqrt{\frac{40 . D_{h}}{U}} \quad \text { Equation } 1
$$

where;

40 is the constant factor for air viscosity $\left({ }^{\circ} \mathrm{C}\right)$

$D h$ is the equivalent hydraulic diameter of the air inlet nozzle(s) $(\mathrm{mm})$

$U$ is the impact velocity $(\mathrm{m} / \mathrm{s})$.

For a circular (sieve) opening, $D h$ is the hole diameter. For a rectangular slit, the equivalent hydraulic diameter will be approximately twice the slit width. Computational fluid dynamics (CFD) has also been shown to be an effective method for the determination of $d 50$ values.

A suitable test method makes use of particles of different sizes, adjusted by the addition of potassium iodide as a bulking agent, containing spores of Bacillus atrophaeus NCTC 10073 or ATCC 9372 strain which survive the collection conditions, including the use of potassium iodide. To obtain the concentrations of spores in the test chamber, a membrane filter (such as cellulose nitrate) is used as a reference sampler. The concentration obtained from the test sampler is compared to the concentration from the membrane filter over several particle sizes, from sub-micron to $15 \mu \mathrm{m}$, over numerous tests, giving an indication of the $50 \%$ cut-off size for the sampler. The physical collection efficiency is calculated using equation 2;

$$
\text { Physical collection efficiency }(\%)=\frac{\text { test sampler count }}{\text { total count from membrane sampler }} \times 100 \quad \text { Equation } 2
$$

\section{Biological Collection Efficiency}

The biological collection efficiency assesses the ability of a sampler to collect viable microbes each of which can form a colony forming unit (cfu). It includes the losses caused by both the physical collection efficiency (the physical loss) and the effect that the sampling has on the viability of the microorganisms (the biological loss) due to stressing during collection and dehydration of the media.

Experimental work to determine the biological collection efficiency requires a test chamber and involves the generation of a suitable test strain of micro-organisms that have a survival rate susceptible to the collection conditions. Staphylococcus epidermidis (NCTC 11047; ATCC 14990) can be used, as this is representative of the (skin) microbial contamination typically found in cleanrooms, and an appropriate reference sampler. Membrane filters are the most physically efficient sampling method available, hence their selection for the determination of the physical collection efficiency. However, Staphylococcus epidermidis is a fragile microbial agent, and after sampling onto a dry, desiccated, (cellulose nitrate) membrane filter, it is unlikely to survive, and so it is an ineffective method for sampling viable microbes. This is not such a problem for the physical efficiency tests that use extremely hardy bacterial spores but is a major issue for the biological collection efficiency testing and an already 
qualified alternative to a membrane sampler, such as an impaction sampler, can therefore be used as a reference. Unlike for the physical tests, bulking agents cannot be utilised to adjust the size of the test aerosol as a high solid content within the bacterial suspension would lead to the bacterial particle embedded within the other solids which could either give an unrealistic protective effect or if potassium iodide was used, would most likely have some inactivation influence. As such the growth suspension of the Staphylococcus epidermidis is diluted in water to make a spray suspension, the water of which evaporates almost instantaneously, leaving a dry (unicellular) particle of a sub-micron size. The biological collection efficiency therefore relates specifically to sub-micron microbes which are not representative of the larger MCPs present in cleanrooms which have an average aerodynamic diameter of about $8 \mu \mathrm{m}$ to $12 \mu \mathrm{m}{ }^{9},{ }^{10},{ }^{11}$. However, as an alternative to the aerosol chamber method, Annex E of BS EN 17141 states that a simplified laboratory method could be carried out in an operational premises so that the tests are performed with naturally occurring micro-carrying particles and not with artificial aerosols. The tests need sufficiently high airborne microbial concentrations to ensure the corresponding number of organisms captured on the growth media provides meaningful data. The sampler under investigation, is compared to a qualified, reference method such as an impaction sampler that has a known good performance. For both methods, the biological collection efficiency is calculated using equation 3;

$$
\text { Biological collection efficiency }(\%)=\frac{\text { test sampler count }}{\text { total count from reference sampler }} \quad \times 100 \quad \text { Equation } 3
$$

\section{Considerations for the 'Biological Efficiency'}

The issue with the testing to determine the biological collection efficiency is that the sub-micron aerosol that is used can be challenging for many test samplers to capture and if the reference sampler used has a lower d50 (cut-off size) than the sampler under test, it could significantly outperform it, due to it being physically more efficient at collecting a sub-micron aerosol. To remove the physical efficiency bias from this test, the sampler 'biological efficiency' is sometimes reported. This is a test chamber method and utilises a mixed sub-micron aerosol of both Staphylococcus epidermidis (white plate colonies) and Bacillus atrophaeus (orange plate colonies) organisms and instead of directly comparing the number of microbes recovered at the same time by the test sampler with those recovered by the reference sampler, the ratio of recovered Staphylococcus epidermidis (Se) and Bacillus atrophaeus (Ba) organisms is determined for both samplers. The 'biological efficiency' is then reported as the Se/Ba ratio for the test sampler compared to the same ratio for the reference sampler without consideration for the physical efficiency of the sampler, which is determined through the physical efficiency tests, across a range of particle sizes. The biological efficiency is determined as shown in equation 4.

$$
\text { Biological efficiency }(\%)=\frac{\text { test sampler ratio Se/Ba }}{\text { reference sampler ratio } \mathrm{Se} / \mathrm{Ba}} \quad \mathrm{x} 100 \quad \text { Equation } 4
$$

It should be noted that the biological efficiency is not required for the sampler validation and is therefore not included in informative Annex E of BS EN 1714. This provides some information regarding the likelihood that any captured microbes would survive, by consideration of the number of sensitive (Se) organisms that are recovered compared to highly resistant (Ba) organisms. However, it does not provide any information regarding the ability of a sampler to actually recover airborne microbial contamination i.e. the biological collection efficiency. For a sampler that recovers very low numbers of microbes, it is likely that it could still present a $(\mathrm{Se} / \mathrm{Ba})$ ratio that, when compared to the same ratio determined for the reference sampler, which actually recovers a much larger number of microbes, will give a reasonably high biological efficiency value. This high 'biological efficiency' is often mistaken for 
the biological collection efficiency and air samplers may be utilised in the false belief that they are appropriate for monitoring well controlled environments where the microbial airborne concentrations are low. However, the testing data can be re-interpreted to provide some information relating to the biological collection efficiency and, if not available, information relating to the physical collection efficiency for sub-micron particles. This is now illustrated by comparing the testing reported for three different air samplers.

\section{Comparison of Testing Data for Different Air Samplers}

Details relating to three different samplers, two slit to agar units with rotating plate heads and a sieve sampler with a fixed plate head are shown in table 1. Also included is the reference Casella sampler, utilised by the testing company. An initial assessment of the performance of each sampler was completed by a review of the air samplers manufacturer's data, following testing of each sampler by the same independent specialist testing company, that reported the 'biological efficiency' for each sampler and also the physical collection efficiency for two of them. The testing was completed according to the methods described in the previous paragraphs and this information is shown in table 2 where it can be seen that testing of the physical collection efficiency was not requested for sampler C. The 'biological efficiencies' (referenced as the 'testing company method' in table 2) have been calculated for all three samplers from the ratios of the total numbers of recovered Staphylococcus epidermidis ( $\mathrm{Se}$ ) and Bacillus atrophaeus ( $\mathrm{Ba}$ ) organisms when compared to the same ratio for the reference sampler. An example of the full testing data completed by the specialist company for the determination of the biological efficiency is shown in table A1 in Annex 1, for air sampler A. Figure 1 Contour plot for time taken for 6 log reduction to be achieved in a capping isolator Table 1 Air samplers evaluated

\begin{tabular}{|c|c|c|c|c|c|c|}
\hline $\begin{array}{c}\text { Air } \\
\text { sampler }\end{array}$ & Description & $\begin{array}{c}\text { Slit/sieve } \\
\text { dimension } \\
(\mathrm{mm})\end{array}$ & $\begin{array}{c}\text { Impact } \\
\text { velocity } \\
(\mathrm{m} / \mathrm{s})\end{array}$ & $\begin{array}{c}D_{50} \\
(\mu \mathrm{m})^{\mathrm{a}}\end{array}$ & $\begin{array}{c}\text { Sampling } \\
\text { flow rate } \\
\text { (1/min) }\end{array}$ & $\begin{array}{l}\text { Time for } \\
1 \mathrm{~m}^{3} \\
\text { sample } \\
\text { (mins) }\end{array}$ \\
\hline A & $\begin{array}{l}\text { Slit to agar (single slit) } \\
\text { Rotating head } \\
140 \text { mm diameter plate } \\
\text { [PinPoint Scientific } \\
\text { ImpactAir - } 140]\end{array}$ & $\begin{array}{c}0.152 \times 44 \\
\text { (width } x \\
\text { length) }\end{array}$ & 72.0 & 0.42 & 28.3 & 35.3 \\
\hline B & $\begin{array}{l}\text { Slit to agar (single slit) } \\
\text { Rotating head } \\
90 \text { mm diameter plate } \\
\text { [PinPoint Scientific } \\
\text { ImpactAir ISOCON-90] }\end{array}$ & $\begin{array}{c}0.200 \times 22 \\
\text { (width x } \\
\text { length) }\end{array}$ & 56.8 & 0.53 & 15.0 & 66.7 \\
\hline $\mathrm{C}$ & $\begin{array}{c}\text { Sieve to agar } \\
179 \text { holes } \\
\text { Fixed head } \\
90 \mathrm{~mm} \text { diameter plate }\end{array}$ & $\begin{array}{c}0.65 \\
\text { (diameter) }\end{array}$ & 10.5 & 1.6 & 50.0 & 20.0 \\
\hline Reference & $\begin{array}{l}\text { Slit to agar (single slit) } \\
\text { Rotating head } \\
90 \text { mm diameter plate } \\
\text { [Casella] }\end{array}$ & $\begin{array}{l}0.300 \times 28 \\
\text { (width } \times \\
\text { length) }\end{array}$ & 59.5 & 0.63 & 30.0 & 33.3 \\
\hline
\end{tabular}

a. At the stated sampling flow rate and calculated according to the method described by Ljungqvist and Reinmüller ${ }^{8}$. 
Table 2 Air sampler performance completed by testing company

\begin{tabular}{|c|c|c|}
\hline Air sampler & $\begin{array}{c}\text { Physical Collection Efficiency } \\
\text { (BS EN 17141) } \\
\text { [Ba Sampler/ } \\
\text { Ba Membrane] } \\
(\%)\end{array}$ & $\begin{array}{c}\text { Biological Efficiency } \\
\text { (Testing company method) } \\
\text { [Air sampler ratio Se:Ba/ } \\
\text { Casella sampler a ratio Se:Ba] } \\
(\%)\end{array}$ \\
\hline A & $\begin{array}{l}0.8 \mu \mathrm{m}-130.7 \\
1.4 \mu \mathrm{m}-123.7 \\
2.8 \mu \mathrm{m}-120.8 \\
6.5 \mu \mathrm{m}-112.2 \\
10.7 \mu \mathrm{m}-99.8 \\
\end{array}$ & 125 \\
\hline B & $\begin{array}{l}0.8 \mu \mathrm{m}-92.9 \\
1.4 \mu \mathrm{m}-100.8 \\
2.5 \mu \mathrm{m}-100.4 \\
6.8 \mu \mathrm{m}-117.8 \\
13.1 \mu \mathrm{m}-119.3 \\
\end{array}$ & 150 \\
\hline $\mathrm{C}$ & Not measured & 183 \\
\hline
\end{tabular}

Ba - Bacillus atrophaeus, Se - Staphylococcus epidermidis

a. Casella is the reference slit to agar sampler utilised by the testing company

\section{Biological and Physical Collection Efficiencies Determined from Testing Data}

It can be seen from table 2 that the 'biological efficiency' has been reported for each sampler but this does not provide information relating to the biological collection efficiency that better determines the suitability of the sampler for use in well controlled cleanrooms.

However, using the testing data, the concentrations of Staphylococcus epidermidis recovered by each sampler can be compared with the reference sampler and the biological collection efficiency, for the sub-micron size of the aerosolised microbes utilised, can be determined. Similarly, the Bacillus atrophaeus concentrations can be utilised to provide some information regarding the physical collection efficiency, which again relates to the size of microbes recovered. Shown in table A2 of Annex 1 , is an example of the accumulated recovered microbial counts derived for sampler $A$ and also for the reference Casella sampler. With a knowledge of the air sampling rates and sampling times and shown in table 1 , the sample volume can be determined and the resultant number of recovered microbes normalised to a $1 \mathrm{~m}^{3}$ volume to enable comparison with the reference sampler. This information is summarised in table 3 for all three test samplers. 
Table 3 Air sampler performance derived from testing data

\begin{tabular}{|c|c|c|c|c|c|c|c|}
\hline \multirow[t]{2}{*}{ Sampler } & \multirow[t]{2}{*}{$\begin{array}{c}\text { Sampling } \\
\text { Rate } \\
\text { (1/min) }\end{array}$} & \multirow[t]{2}{*}{$\begin{array}{c}\text { Sampling } \\
\text { Time } \\
\text { (min) }\end{array}$} & \multicolumn{2}{|c|}{$\begin{array}{c}\text { Average } \\
\text { Microbial } \\
\text { Concentrations } \\
\left(\mathbf{c f u} / \mathbf{m}^{3}\right)\end{array}$} & \multirow[t]{2}{*}{$\begin{array}{l}\text { Ratio } \\
\text { Se/Ba }\end{array}$} & \multirow{2}{*}{$\begin{array}{c}\text { Biological Collection } \\
\text { Efficiency } \\
\text { (EN 17141) } \\
\text { [Se Sampler/ } \\
\text { Se Casella"] } \\
(\%)\end{array}$} & \multirow{2}{*}{$\begin{array}{c}\text { Physical Collection } \\
\text { Efficiency } \\
\text { (EN 17141 Based) } \\
\text { [Ba Sampler/ } \\
\left.\text { Ba Casella }{ }^{2}\right] \\
(\%)\end{array}$} \\
\hline & & & $\mathbf{B a}$ & Se & & & \\
\hline A & 30 & 2 & 2620 & 1732 & 0.66 & 254 & 228 \\
\hline Casella $^{\mathbf{a}}$ & 30 & 2 & 1174 & 681 & 0.58 & - & - \\
\hline $\mathrm{B}$ & 15 & 2 & 1069 & 5969 & 5.58 & 226 & 159 \\
\hline Casella $^{a}$ & 30 & 2 & 671 & 2673 & 3.98 & - & - \\
\hline $\mathrm{C}$ & 50 & 2 & 321 & 1147 & 3.58 & 7.3 & 5.3 \\
\hline Casella $^{a}$ & 30 & 0.5 & 6060 & 15700 & 2.59 & - & - \\
\hline
\end{tabular}

Ba - Bacillus atrophaeus, Se - Staphylococcus epidermidis

a. Casella is the reference slit to agar sampler utilised by the testing company

\section{Discussion of Testing Results}

It can be seen from table 1 that sampler $C$ has the lowest impact air velocity and hence the lowest $\mathrm{d} 50$ value which is significantly lower than the corresponding value for samplers $A$ and $B$. As the physical collection efficiency of sampler $C$ was not requested from the testing company, it is reasonable to assume that, with considerations of the physical collection efficiencies determined for samplers $A$ and B shown in table 2, if tested for sampler C, would have been found to have a low value. This is supported by the testing data shown in table 3 for sampler $\mathrm{C}$ where it can be seen that despite a higher sampling rate $(50 \mathrm{l} / \mathrm{min})$ compared with the reference Casella sampler $(30 \mathrm{l} / \mathrm{min})$, a sampling time (2 $\mathrm{min}$ ) that was 4 times greater than that utilised for the reference sampler $(0.5 \mathrm{~min})$ was required in order for it to capture sufficient microbes. Even under these sampling conditions, the resultant number of recovered microbes was greatly reduced compared with the number recovered by the reference sampler. However, similarly for samplers A and B, with the sampling time the same as that used for the reference sampler, the recovered numbers were actually much higher than recovered by the reference sampler. Consequently, when the recovered numbers of microbes were utilised to determine the biological collection efficiency and also provide some information regarding the physical collection efficiency, both of these values for sampler $\mathrm{C}$ were found to be $7.3 \%$ and $5.3 \%$ respectively and therefore extremely low when compared to the reference sampler. In contrast, and as anticipated from the information shown in table 1 , the corresponding values determined for samplers $A$ and $B$ were $254 \%$ and $226 \%$ respectively for the biological collection efficiency and $228 \%$ and $159 \%$ respectively for the physical collection efficiency. A review of table 2 however indicates that sampler $C$ had the highest reported 'biological efficiency' value (183\%) compared to samplers A and B $(125 \%$ and $150 \%$ respectively) and if the 'biological efficiency' was considered to be indicative of air sampler performance, such samplers may be used in the false belief that they would be suitable for monitoring within well controlled cleanrooms.

The overall effectiveness of a microbial air sampler must therefore be determined from a review of the both the physical and biological collection efficiency test results. If the sampler has a high biological collection efficiency (measured for microbes of sub-micron size), then by review of the physical collection data, an assessment can be made regarding the likely biological collection efficiency for larger MCPs found within cleanroom environments. This is shown in table 2 for samplers A and B where it can be seen that the physical collection efficiencies remain reasonably consistent across the range 
of particle sizes tested and it is considered that the biological collection efficiency would be retained for the collection of larger MCPs.

However, a further consideration relates to the sampler impact velocity of the sampled air and the functionality of the sampler head, which may remain static or can have speed controlled rotation, on which the media plate is located. A fixed head sieve sampler that has a high impaction velocity may also have a correspondingly high physical collection efficiency but it may have a poor biological collection efficiency. This is because the microbes captured onto the static agar surface are continuously exposed to the desiccating effects of the sampler airflow at the point of impaction throughout the entire sampling process. Conversely, many sieve type samplers that use lower impaction velocities have very poor physical collection efficiencies at small particle sizes, but due to the associated less aggressive collection process, are more likely to retain the viability of the captured microbes. Slit samplers that have rotating heads are effective in offsetting the effect of the high impaction velocities as the captured microbes are moved away from the impaction zone, reducing the effects of the agar desiccation. Consequently, slit samplers with high impact velocities that also utilise rotating head technology are the preferred option as microbial air samplers.

The test method outlined in BS EN 17141 to determine the physical collection efficiency is able to utilise resistant microbes, associated with various sizes of particles, the collection of which are evaluated relative to a reference membrane sampler. Unfortunately, this robust testing approach that provides information for microbe-carrying particles of various sizes, relative to a definitive reference device, cannot be utilised for the biological efficiency testing. In this case, another air sampler needs to be utilised as the reference instrument due to the fragile nature of the test organism that will be compromised by impaction onto a membrane filter and also the need to test with aerosols that provide sub-micron size test organisms. The use of another air sampler as a reference instrument presents significant issues as different testing companies may not utilise the same reference sampler making direct comparisons between tested air samplers difficult. The use of sub-micron test organisms also presents significant issues, as the performance cannot be determined directly for larger MCPs and an indirect assessment needs to be made utilising the physical efficiency testing information. If the physical testing is not available, this may therefore lead to the introduction of other reported testing parameters, such as the 'biological efficiency', that may present misleading information regarding the suitability for use of the sampler for the intended application. Significant improvements to the test could therefore be made if a standard reference device (not another air sampler) that does not significantly compromise the recovery of the captured microbes could be utilised. A gelatine type filter, that has a soft, moist surface could provide such a suitable test medium and is readily available and relatively simple to use. Furthermore, if the testing could also be completed in a suitable environment, under more normal conditions using naturally occurring airborne MCPs, a direct measurement of real life performance would be possible, avoiding the need for the characterization of efficiency against particle size, obtained from the physical collection efficiency tests.

In order to provide effective microbial monitoring, the sampling should be completed in locations where the risk of contamination of product, critical surfaces or the environment is greatest. The air sampler used must have an appropriate and verified performance that can be determined by consideration of both the physical and biological collection efficiencies which are derived experimentally. The physical collection efficiency testing provides robust information, as it is able to utilise resistant microbes, of various particle sizes, the collection of which are evaluated relative to a constant reference membrane sampler. The physical testing needs to be completed because the biological efficiency testing is not as robust and can only be determined for sub-micron sized microbes, that are not representative of the larger size of microbe-carrying particles typically present within 
cleanrooms. Consequently, the physical testing results then need to be reviewed to indirectly determine the likely biological collection efficiency for the larger microbe-carrying particles. If the physical efficiency testing is not completed, other testing parameters, such as the 'biological efficiency', may be reported that may present misleading information regarding the performance of the sampler for the intended application.

It is therefore concluded that if improvements to the biological collection efficiency test method could be made, significant enhancement to the effectiveness of the sampler performance evaluation process would be achieved. It is recommended that the test procedure investigates the possibility of the utilisation of a constant reference sampler, such as a gelatine membrane filter (instead of a microbial air sampler) that does not have a significant effect on the viability of the collect microbes. Furthermore, consideration should be given to completing the testing in an environment using naturally occurring airborne MCPs, so that a direct measurement of real life performance would be possible. 


\section{REFERENCES}

01. The rules governing medicinal products in the European Union -Volume 4 EU guidelines to good

manufacturing practice - medicinal products for human and veterinary use - Annex 1 -Manufacture of sterile medicinal products. European Commission, Brussels, 2009.

02. Eaton T, Whyte W. Pharmaceutical Cleanroom Classification using ISO 14644 and the EU GGMP Annex 1. Part 1: Testing rationale. European Journal of Parenteral and Pharmaceutical Sciences 2019; 24(4).

03. Eaton T, Whyte W. Pharmaceutical Cleanroom Classification using ISO 14644 and the EU GGMP Annex 1; Part 2: Practical application. European Journal of Parenteral and Pharmaceutical Sciences 2019; 24(4).

04. W. Whyte, G. Green, A. Albisu. Collection efficiency and design of microbial air samplers. Aerosol Science 38 (2007) $101-114$

05. BS EN 17141: 2020 Cleanrooms and associated controlled environments -Biocontamination Control. The British Standards Institution, 2020

06. ISO 14698-1: 2003 Cleanrooms and associated controlled environments-Biocontamination control, Part 1: General principles and methods. Geneva, Switzerland, International Organization for Standardization, 2003.

07. ISO 14698-2: 2003 Cleanrooms and associated controlled environments-Biocontamination control, Part 2: Evaluation and interpretation of biocontamination data. Geneva, Switzerland, International Organization for Standardization, 2003

08. Ljungqvist B and Reinmüller B. Active sampling of airborne viable particles in controlled environments: a comparative study of common instruments. European Journal of Parenteral Sciences 1998; 3:59-62.

09. T Eaton, C Davenport and W Whyte. Airborne microbial monitoring in an operational cleanroom using an instantaneous detection system and high efficiency microbiological samplers. European Journal of Parenteral \& Pharmaceutical Sciences 2012; 17(2): 61-69.

10. Noble WC, Lidwell OM and Kingston D. The size distribution of airborne particles carrying microorganisms. Journal of Hygiene 1963; 61: 385-391.

11. Whyte $W$ and Hejab M Particle and microbial airborne dispersion from people. European Journal of Parenteral and Pharmaceutical Science 2007; 12(2): 39-46. 
ANNEX

Example Testing Information for Sampler A, PinPoint Scientific ImpactAir -140

Table A1 Air Sampler Testing Results, Biological Efficiency

\begin{tabular}{|c|c|c|c|c|c|c|c|}
\hline \multirow{3}{*}{ TEST } & \multicolumn{3}{|c|}{ Casella (reference sampler) } & \multicolumn{3}{|c|}{ ImpactAir -140 } & \multirow{3}{*}{$\begin{array}{c}\text { Biological Efficiency } \\
\text { ImpactAir-140/ } \\
\text { Casella } \\
(\%)\end{array}$} \\
\hline & cfu & cfu & ratio & cfu & cfu & ratio & \\
\hline & $\mathrm{Ba}$ & $\mathrm{Se}$ & $\mathrm{Se} / \mathrm{Ba}$ & $\mathrm{Ba}$ & $\mathrm{Se}$ & $\mathrm{Se} / \mathrm{Ba}$ & \\
\hline 1 & 73 & 30 & 0.41 & 160 & 75 & 0.47 & 114.06 \\
\hline 2 & 50 & 45 & 0.90 & 96 & 117 & 1.22 & 135.42 \\
\hline 3 & 36 & 39 & 1.08 & 110 & 99 & 0.90 & 83.08 \\
\hline 4 & 29 & 36 & 1.24 & 46 & 100 & 2.17 & 175.12 \\
\hline 5 & 14 & 33 & 2.36 & 45 & 102 & 2.27 & 96.16 \\
\hline 6 & 124 & 65 & 0.52 & 354 & 147 & 0.42 & 79.22 \\
\hline 7 & 98 & 48 & 0.49 & 278 & 124 & 0.45 & 91.07 \\
\hline 8 & 74 & 45 & 0.61 & 232 & 128 & 0.55 & 90.73 \\
\hline 9 & 70 & 55 & 0.79 & 223 & 139 & 0.62 & 79.33 \\
\hline 10 & 125 & 47 & 0.38 & 293 & 103 & 0.35 & 93.49 \\
\hline 11 & 93 & 51 & 0.55 & 188 & 123 & 0.65 & 119.31 \\
\hline 12 & 88 & 38 & 0.43 & 195 & 81 & 0.42 & 96.19 \\
\hline 13 & 67 & 31 & 0.46 & 117 & 96 & 0.82 & 177.34 \\
\hline 14 & 76 & 34 & 0.45 & 156 & 115 & 0.74 & 164.78 \\
\hline 15 & 71 & 38 & 0.54 & 137 & 83 & 0.61 & 113.20 \\
\hline 16 & 66 & 39 & 0.59 & 82 & 111 & 1.35 & 229.08 \\
\hline 17 & 80 & 34 & 0.43 & 120 & 73 & 0.61 & 143.14 \\
\hline 18 & 56 & 31 & 0.55 & 84 & 86 & 1.02 & 184.95 \\
\hline 19 & 43 & 37 & 0.86 & 71 & 72 & 1.01 & 117.85 \\
\hline & & & & & & Average & 125.45 \\
\hline & & & & & & Deviation & 42.79 \\
\hline
\end{tabular}

Ba - Bacillus atrophaeus, Se - Staphylococcus epidermidis

Table A2 Calculation of Microbial Counts per $\mathrm{m}^{3}$

\begin{tabular}{|l|c|c|c|c|}
\hline \multicolumn{1}{|c|}{ Sampling } & \multicolumn{2}{c|}{ Casella } & \multicolumn{2}{c|}{ ImpactAir-140 } \\
\hline Sample rate (L/min) & \multicolumn{2}{|c|}{30} & \multicolumn{2}{c|}{30} \\
\hline Sample time (min) & \multicolumn{2}{c|}{2} & \multicolumn{2}{c|}{2} \\
\hline Sample volume (L) & \multicolumn{2}{|c|}{60} & \multicolumn{2}{c|}{1140} \\
\hline Total sample volume (L) & \multicolumn{2}{|c|}{1140} & Ba & Se \\
\hline \multicolumn{1}{|c|}{ Recovered CFU } & Ba & Se & 3040 & 1974 \\
\hline Total count & 1333 & 776 & 2667 & 1732 \\
\hline Counts/ $\mathrm{m}^{3}$ & 1169 & 681 & \\
\hline
\end{tabular}

Ba - Bacillus atrophaeus, Se - Staphylococcus epidermidis 